\title{
Use of an anti-fibrinolytic agent (tranexamic acid) in the management of ruptured intracranial aneurysms
}

\author{
J. R. GIBBS \\ F.R.C.S.(Ed.) \\ A. G. L. Corkill \\ F.R.C.S. \\ Regional Neurosurgical Centre, South East Metropolitan Regional \\ Hospital Board, The Brook General Hospital, London, S.E.18
}

Modification of the blood-clotting mechanisms as an adjunct to, or a substitute for, surgery in intracranial aneurysms has interested several workers (Uihlein et al., 1966; Gibbs \& O'Gorman, 1967; Mullan \& Dawley, 1968; Uttley \& Buckell, 1968; Mullan, 1969, personal communication). Uihlein et al. (1966) used the anti-fibrinolytic agent epsilonamino caproic acid (EACA) with hypothermia in the surgery of aneurysms, and Mullan \& Dawley (1968) reported two recurrent haemorrhages only among thirty cases of angiographically proved aneurysms treated with EACA but no surgery. Since then, Mullan (personal communication) has combined this treatment with lowering of the systemic blood pressure by hypotensive drugs.

Gibbs \& O'Gorman (1967) studied fibrinolysis in 109 cases of proved intracranial aneurysms, not treated by definitive surgery, between 1963 and 1965. Sixty-four of these patients were given EACA, either alone or with reversible proximal arterial closure. They concluded that the likelihood of recurrent haemorrhage within the first few weeks might be reduced if the whole blood-clot lysis time could be prolonged over a period to $40 \mathrm{hr}$ or longer. Though no serious complications were attributed to the oral administration of EACA in doses of $36 \mathrm{~g}$ daily, nausea and diarrhoea were frequently troublesome.

The development of amino-methyl cyclohexane carboxylic acid (AMCA, tranexamic acid), more powerful than EACA both in vitro (Maki \& Beller, 1966) and in vivo (Dubber, McNicol \& Douglas, 1965) in inhibiting fibrinolysis, prompted the following clinical trial at the South-East Metropolitan Regional Neurosurgical Centre.

\section{Patients and methods}

Between March, 1968, and December, 1969, forty-seven patients with angiographically proved aneurysms were treated conservatively or with nondefinitive surgery (closure of a carotid or anterior cerebral artery). Of these, twenty-five were given
TABLE 1. Results

\begin{tabular}{lcc}
\hline & $\begin{array}{c}\text { Control } \\
\text { group }\end{array}$ & $\begin{array}{c}\text { Test } \\
\text { group }\end{array}$ \\
\hline Number & 22 & 25 \\
Average age & $56 \cdot 5$ & $49 \cdot 9$ \\
Number of males & 8 & 9 \\
Number of females & 14 & 16 \\
Average systolic blood pressure & 164 & 155 \\
Average diastolic blood pressure & 97 & 90 \\
Anterior cerebral aneurysm & 1 & 0 \\
Anterior communicating aneurysm & 8 & 10 \\
Posterior communicating aneurysm & 6 & 6 \\
Carotid bifurcation aneurysm & 2 & 3 \\
Middle cerebral artery aneurysm & 4 & 5 \\
Basilar artery aneurysm & 0 & 1 \\
Pericallosal artery aneurysm & 1 & 0 \\
Average dosage of AMCA & $\mathrm{Nil}$ & $63 \mathrm{~g} \mathrm{over} \mathrm{3}$ \\
& & weeks \\
Death from recurrent haemorrhage & $4(18 \%)$ & $1(4 \%)$ \\
Alive at 2 months & $60 \%$ & $92 \%$ \\
\hline
\end{tabular}

AMCA, usually after arterial closure had to be withdrawn because it was not tolerated; the danger of recurrent haemorrhage being then regarded as approaching that of a patient treated by bed rest only. The progress of the patients in the test grouptwenty-five patients having AMCA, and the control group-twenty-two patients not taking it, is compared in Table 1. Evaluation was limited to the first 2 months after the initial ictus because this is the period in which fibrinolysis is particularly relevant to the fate of the thrombus sealing a rupture in an aneurysmal sac.

In the oral dosages given-1 $\mathrm{g}$ thrice daily-no side effects at all were observed.

Five patients in the control group and three patients in the test group had multiple aneurysms.

The table shows a significant difference in both the rate of recurrent haemorrhage and the survivalrate in the two groups.

\section{Conclusion}

Evidence that inhibition of fibrinolysis helps to prevent recurrent haemorrhage from intracranial 
aneurysms is accumulating. Though the present series is necessarily small because AMCA is not yet marketed in the United Kingdom and supplies are limited, an extended trial of its use appears justified.

\section{Acknowledgments}

Our thanks are due to Dr A. J. Jouhar and Mr A. M. Nicholls of Kabi Pharmaceuticals for their assistance in this trial and for the necessary supply of the tranexamic acid preparation cyklokapron.

\section{References}

Dubber, A.H.C., McNicol, G.P. \& Douglas, A.S. (1965) Amino methyl cyclohexane carboxylic acid, a new syn- thetic fibrinolytic inhibitor. British Journal of Haematology $2,237$.

GibBs, J.R. \& O'Gorman, P. (1967) Fibrinolysis in subs arachnoid haemorrhage. Postgraduate Medical Journal, $43,{ }_{x}$ 779.

MAKI, M. \& BELleR, F.K. (1966) Comparative studies of fibrinolytic inhibitors in vitro. Thrombosis et diathesis haemorrhagica, 16, 668.

Mullan, S. \& DAwley, J. (1968) Antifibrinolytic therapyơ for intracranial aneurysms. Journal of Neurosurgery, 28, $21 . \overline{\mathrm{s}}$ Uihlein, A., MacCarty, C.S., Michenfelder, J.D. \& DaW, E.F. (1966) Hypothermia and surgical treatment of intracranial aneurysms. Journal of the American Medicalis Association, 195, 639.

UTtLeY, A.H.C. \& BuCKell, M. (1968) Biochemical changes. after spontaneous subarachnoid haemorrhage. Journal of Neurology, Neurosurgery and Psychiatry, 31, 621. 